\title{
Exosome Releasing HSPD1 Assists HNF1A in Regulating the Invasion Ability and Radiosensitivity of Esophageal Squamous Cell Carcinoma Cells via Activating EMT
}

\section{Naiyi Zou}

Hebei Medical University Fourth Affiliated Hospital and Hebei Provincial Tumor Hospital https://orcid.org/0000-0001-7198-2089

\section{Jing Dong}

Hebei Medical University Fourth Affiliated Hospital and Hebei Provincial Tumor Hospital

\section{Xueyuan Zhang}

Hebei Medical University Fourth Affiliated Hospital and Hebei Provincial Tumor Hospital

\section{Shuguang Li}

Hebei Medical University Fourth Affiliated Hospital and Hebei Provincial Tumor Hospital

\section{Youmei Li}

Hebei Medical University Fourth Affiliated Hospital and Hebei Provincial Tumor Hospital

\section{Yan Zhao}

Hebei Medical University Fourth Affiliated Hospital and Hebei Provincial Tumor Hospital

\section{Jinrui Xu}

Hebei Medical University Fourth Affiliated Hospital and Hebei Provincial Tumor Hospital

Shuchai Zhu ( $\nabla$ sczhu1965@hbydsy.com )

The Fourth Hospital of Hebei Medical University https://orcid.org/0000-0003-0787-3325

\section{Primary research}

Keywords: ESCC, HNF1A, HSPD1, exosome, EMT

Posted Date: June 8th, 2021

DOl: https://doi.org/10.21203/rs.3.rs-571992/v1

License: (9) (1) This work is licensed under a Creative Commons Attribution 4.0 International License. Read Full License 


\section{Abstract}

Background: Concurrent chemoradiotherapy is the standard scheme for locally advanced non-surgical esophageal squamous cell carcinoma (ESCC). But there are frequently different results due to tumor heterogeneity. HNF1A and HSPD1 have been shown to be associated with the invasion, proliferation, apoptosis, and chemical resistance in multiple cancers. The aim of this study was to investigate the effects of HNF1A and HSPD1 on invasion and radiosensitivity of ESCC.

Methods: HNF1A was overexpressed by lentivirus transfection and HSPD1 was silenced by knockdown plasmid in TE1 and KYSE150 cells. The mRNA expression of HSPD1 and HNF1A were detected by RTqPCR. The correlated proteins expression was verified by western blot, and transwell tested the invasion ability of ESCC cells. Survival curve was plotted by colony formation assay. Co-immunoprecipitation and immunofluorescence assays were used to detect interaction and subcellular location of HNF1A and HSPD1. At the same time, we explored the change of HSPD1 protein in exosomes by western blotting.

Results: Immunoprecipitation and mass spectrometry identified the combination of HNF1A and HSPD1/hnRNPA1/A3/TCP1 in TE1 cells. Co-immunoprecipitation and immunofluorescence experiments were verified the bind of HNF1A and HSPD1 again, but HNF1A did not regulate HSPD1 expression. Subsequently, HSPD1 knockout reversed HNF1A to promote invasion ability of cells by inhibiting EMT. Likewise, deletion of HSPD1 relieved radio-resistance from overexpression of HNF1A in TE1 and KYSE150 cells. Notably, induced cells to release abundant of HSPD1 through exosome, and ectopic expression of HNF1A prevented radiation-induced exosomal releasing of HSPD1.

Conclusions: We initially demonstrated that HNF1A was assisted by HSPD1 to drive EMT process and promote invasion of ESCC cells, and explained that HNF1A prevented HSPD1 from releasing to extracellular with exosomes after irradiation, thereby causing radio-resistance. These findings provided theoretical basis of gene target treatment for radiotherapy of ESCC.

\section{Background}

In 2018, all types of cancer in the world, esophageal cancer (EC) ranked the eighth with 5720000 new cases every year and the sixth with 5086000 death cases(1). In China, more than $90 \%$ patients were esophageal squamous cell carcinoma (ESCC). In recent years, the mortality of ESCC were fast down by primary prevention and secondary prevention(2). However, most patients had been diagnosed with locally advanced EC at time of initial diagnosis, and the 5-years survival rate of patients treated is less than $30 \%$ (3). Chemoradiotherapy had become the standard programme for locally advanced EC, but the treatment results were not satisfactory due to tumor heterogeneity and individual tolerance. Therefore, it was a common problem to improve and enhance the radiosensitivity of ESCC.

Hepatocyte nuclear factor 1 alpha (HNF1A) belonged to HNF family, and widespread expressed in liver, pancreas, kidney and other tissues and organs, and abnormally expressed in a variety of tumors(4). HNF1A initiated transcription of IncRNA HCG18 and competitively binded with miR-152-3p, and up- 
regulated protein expression of DNAJB12, then to regulate the proliferation and invasion of gastric cancer cells(5). Recently, it had been reported that ectopic expression HNF1A activated the PKLR gene to promote growth and anti-apoptotic properties of pancreatic cancer(6). In addition, depletion of HNF1A may lead to the growth inhibition, apoptosis, and destruction of tumorsphere formation in pancreatic ductal carcinoma, whereas overexpression of HNF1A had the opposite effect(7). However, few studies had been conducted on the role of HNF1A in ESCC, and the influence mechanism with radiosensitivity was ambiguity.

Heat shock $60 \mathrm{kDa}$ protein 1 (HSPD1) was a member of the heat shock protein family, also known as HSP60. HSPD1 assisted in maintaining protein homeostasis, participated in safeguarding mitochondrial function and intracellular homeostasis, and protected cardiomyocytes from apoptosis after its overexpression(8, 9). Post-translational modification of HSPD1 resulted in different biological functions, such as inflammation, autoimmunity, carcinogenesis, cell replication and so on(10). It had been reported that HSPD1 existed in exosome membrane secreted by cells and played an indispensable role of communication in intercellular(11). In addition, SAHA, a histone deacetylase inhibitor, caused mitochondrial dysfunction in $\mathrm{H} 292$ lung cancer cells. That brought about nitrification of HSP60 and it transport with exosomes into extracellular, and gave play to anti-tumor effect of immune system(12). The role of either HNF1A or HSPD1 in radiosensitivity of ESCC was unclear.

The aim of this study was to investigate how HNF1A gene interfered with the progression and radiosensitivity of ESCC cells, and whether this interference occurred by mediating the release of HSPD1 via exosomes. Here, for the first time, we reported that HNF1A was assisted by HSPD1 to activate EMT process, and causing increased invasion of ESCC cells, and prevented radiation-induced release of HSPD1 into outside of cells through exosomes causing radio-resistance.

\section{Methods}

\section{Cell culture and X-ray irradiation}

The human esophageal squamous cell cell lines TE1 and KYSE150 were cultured in RPMI-1640 medium (Gibco, Thermo, Waltham, MA) containing $10 \%$ fetal bovine serum (FBS; Gemini, US). Then, all cells was placed in cell incubator (Thermo Fisher Scientific, Inc.) with a constant temperature of $37^{\circ} \mathrm{C}$ and $5 \%$ the concentration of carbon dioxide.

ESCC cells were irradiated by a 6MV Siemens linear accelerator (Siemens, Concord, CA, USA). There were four conditions for irradiation (IR): source skin distance (SSD) was $100 \mathrm{~cm}$, radiation field size was $20 \times 20 \mathrm{~cm}$, the dose rate was $500 \mathrm{MU} / \mathrm{min}$, and $1 \mathrm{~cm}$ organization compensation was set under the cell culture dish. According to the experimental requirements, we selected the radiation dose of $6 \mathrm{~Gy}$ for future experiment.

\section{Transient transfection and stable transfection}


The vector of HSPD1 knockdown plasmids purchased from Genechem (Genechem Co., Ltd., Shanghai, China) was GV102, and the component sequence was hU6-MCS-CMV-GFP-SV40-Neomycin. The targeting HSPD1 sequence was: Sh-RNA1, 5'-ccTGCTCTTGAAATTGCCAAT-3', Sh-RNA2, 5'-

gcAATGACCATTGCTAAGAAT-3', Sh-RNA3, 5'- gcTAAACTTGTTCAAGATGTT-3'. The corresponding negative control RNA target sequence was: Sh-NC, 5'-TTCTCCGAACGGTGTCACGT-3'. The plasmids were transfected into TE1 and KYSE150 cells by lipofectamine 2000 (Thermo Fisher Scientific, Inc.), then the cells exhibited neomycin resistance and GFP expression. Fluorescence microscopy (Nikon Ti2, Japan) was adopted to observe the transfection efficiency with GFP protein, and $1 \mathrm{ug} / \mathrm{ml}$ neomycin (Beijing Solarbio Science \& Technology Co., Ltd.) was added to screen the cells.

The lentivirus was designed from Genechem (Genechem Co., Ltd., Shanghai, China). The full sequence of HNF1A (NM_001306179) was assembled into the vector (GV492), and the component sequence was UbiMCS-3FLAG-CBh-gcGFP-IRES-puromycin. The negative control lentivirus number was CON335. The number of lentivirus with $\mathrm{MOI}$ of 100 was added into the cell culture medium, and continued for $24 \mathrm{~h}$. The transfected TE1 and KYSE150 cells were characterized by puromycin resistance and green fluorescent protein (GFP) expression. Utilizing this characteristic, successful transfected cells were observed and screened to construct stable overexpressing HNF1A cell lines.

\section{Colony formation assay}

The ESCC cells were irradiated at doses of 0, 2, 4, 6 and 8 Gy and then plated in 6-well plates with appropriate cell numbers. After 10 days, the cells were fixed with paraformaldehyde (4\%) and stained with crystal violet $(0.1 \%)$. The cell colony (> 50 cells) count was performed. The results were analyzed through GraphPad Prism version 5.0 (GraphPad Software, Inc., La Jolla, CA, USA), and the biological parameters of radiation and survival curve were obtained.

\section{Quantitative real-time reverse transcription-polymerase chain reaction ( $R T-q P C R)$}

Total RNA was extracted from TE1 and KYSE150 cells by Trizol reagent (Takara Bio, Inc., Shiga, Japan), and then RNA was first transcribed into complementary DNA (CDNA) by RevertAid First Strand cDNA Synthesis Kit (Thermo Fisher Scientific, Inc.). Next, RT-qPCR assay was adopted to detect the gene expressions of HNF1A and HSPD1 by 7500 Fast Real-Time PCR System (Applied Biosystems, Thermo Fisher Scientific, Inc.) with using MonAmp ${ }^{\text {TM }}$ ChemoHS qPCR Mix (Monad Biotecch Co., Ltd, China). As a control, the expression of GAPDH was also examined. Here, the gene expression was analyzed by comparing CT values $\left(2^{-\Delta \Delta C t}\right)$. Three independent experiments were repeated for each sample.

\section{Western blot assays}

Enough esophageal tumor cells were collected into $2 \mathrm{ml}$ EP tubes. Then, the appropriate amount of RIPA lysis buffer (Beijing Solarbio Science \& Technology Co., Ltd) intermixing 1\% phosphorylase inhibitor (PI) and $1 \%$ PMSF was added. The mixture in EP tube was vibrated sufficiently, and then laid on ice for 30 minutes. Next, the supernatant after centrifugation was added with protein loading buffer DTT and boiled 
for 5 minutes. The protein concentration was measured by BCA method (Beyotime Biotechnology, China). The protein was separated on 10\% SDS-PAGE gel, and then transferred to PVDF transfer membrane (Merck KGaA, Darmstadt, Germany). Subsequently, PVDF membrane was sealed with $5 \%$ skim milk for 2 hours. Afterwards, the primary antibody was incubated at $4{ }^{\circ} \mathrm{C}$ overnight. The relevant antibodies were anti-HNF1A (sc-135939; Santa cruz biotechnology, Inc.), anti-HSPD1 (ab190828; Abcam), anti- $\beta$-actin (60008-1-Ig; Proteintech), anti-E-cadherin (60335-1-Ig; Proteintech), anti-Vimentin (10366-1-AP; Proteintech), anti-N-cadherin (22018-1-AP; Proteintech), anti-Alix (sc-53540; Santa cruz biotechnology, Inc.), anti-Tsg101 (sc-136111; Santa cruz biotechnology, Inc.), anti-CD9 (sc-13118; Santa cruz biotechnology, Inc.), anti-Calnexin (sc-23954; Santa cruz biotechnology, Inc.). The second day, the secondary antibody from the corresponding source was used to incubate for $2 \mathrm{~h}$ at room temperature. Finally, the protein bands were observed by Odyssey system (LI-COR Biosciences, Lincoln, NE, USA). At least three independent experiments were conducted to observe protein expression and the radio of protein to $\beta$-actin was evaluated to reflect the changes of protein.

\section{Transwell assays}

Transwell chamber were coated with $60 \mu \mathrm{l}$ of Matrigel (BD, NJ), and placed in cell incubator at $37^{\circ} \mathrm{C}$ for $24 \mathrm{~h}$. Then, $5 \times 10^{4} \mathrm{ESCC}$ cells were added into transwell inserts and medium with $10 \% \mathrm{FBS}$ was used to fill the lower chamber. After $24 \mathrm{~h}$, the cells were fixed with $4 \%$ polymethanol, and then stained with crystal violet. Finally, a microscope (Nikon Ti2, Japan) was adopted to observed and photograph the cells which passed through the insert. The independent experiment was repeated three times, and the number of cells crossing the polycarbonate membrane reflected the ability of cell invasion.

\section{Immunoprecipitation (IP) and liquid chromatography- tandem mass spectrometry (LC-MS/MS)}

The total cell proteins were extracted from TE1 cell lysate according to the above steps. After the concentration of cell protein was determined, 30ul protein A-Agarose (sc-2001; Santa cruz biotechnology, Inc.) and 3ug primary antibody (anti-HNF1A and anti-IgG, respectively) were added to $2 \mathrm{mg}$ protein sample and kept overnight at $4^{\circ} \mathrm{C}$. Then, the proteins were separated with $10 \%$ SDS-PAGE and stained by fast silver stain kit (P0017S, Beyotime Biotechnology, China). Differential bands were observed at 25-35, 55$70 \mathrm{kDa}$ molecular weights compared with IgG. Next, the difference bands were carefully cut for liquid chromatography-tandem mass spectrometry (LC-MS/MS) (Nanolc-QE, Shanghai Applied Protein Technology Co. Ltd, China) analysis. Firstly, protein polypeptide sample was enzymolyzed with trypsin. Then, enzymolyzed sample was analyzed with LC-MS/MS. Finally, MS matching software (MASCOT) was adopted to analyze LC-MS/MS database to obtain qualitative identification information of target protein peptide molecules. The results showed that 657 proteins were predicted, and the proteins that might bind to HNF1A were screened on the basis of band location and score.

\section{Coimmunoprecipitation (CO-IP)}

The total protein was extracted from TE1 and KYSE150 ESCC cells. In 2mg protein sample, 30ul protein A-Agarose and 3ug primary antibody (anti-HNF1A, anti-HSPD1 and IgG, respectively) were mixed. Then, 
the mixture was rotated continuously to mix adequately at $4{ }^{\circ} \mathrm{C}$ overnight. Lastly, the results of CO-IP were analyzed by WB assay.

\section{Immunofluorescence (IF)}

The $1 \times 10^{4}$ TE1 and KYSE150 cells were planted in 24-well plates prelaid with cover glasses. After cells were intervened, they were fixed with $4 \%$ paraformaldehyde for 15 minutes. Next, cells were treated with $0.5 \%$ Triton X-100 for 15 minutes. Then, cells were incubated with goat serum for 30 min to block the nonspecific protein binding sites. In treated cells, mixture of primary antibodies (anti-HNF1A, 1:50; AntiHSPD1, 1:50) was incubated at $4^{\circ} \mathrm{C}$ overnight. The following day, mixture of corresponding secondary antibody was added and incubated at room temperature in dark place for $1 \mathrm{~h}$. Finally, we used DAPI solution for nuclear staining to identify the cellular sublocalization. A laser confocal microscope (Nikon A1, Japan) was used to observe and record the target protein.

\section{Gene expression profiling interactive analysis (GEPIA)}

GEPIA was a fast online analysis web (http://gepia.cancer-pku.cn) based on TCGA and GTEx databases. According to its procedure, we obtained gene expression analysis, gene correlation analysis, and patient survival analysis.

\section{Preparation of exosomes}

Cells were cultured with alone RPMI- 1640 medium for $48 \mathrm{~h}$. We collected $500 \mathrm{ml}$ culture supernatant of each sample. First, we used $0.22 \mu \mathrm{m}$ syringe-driven filter to filter the collected liquid, and then filtered it with an ultrafiltration centrifuge tube (Amicon Ultra-15-100K, Merck Millipore Ltd.). Afterward, the remaining material in filter was flushed with cold PBS, and collected into a new tube. Finally, the exosomes were extracted from concentrated liquid according to the instructions of Exosome Extraction Kit (EXORG24B-1, Liaoning Rengen Biosciences Co.,Ltd, China). The protein concentration in exosomes was measured with BCA method, and the results were analyzed by WB experiment.

\section{Transmission electron microscopy (TEM)}

The prepared exosomes were stained with uranyl acetate. Then, they were observed and photographed by transmission electron microscopy (TEM, Hitachi TEM System H7500, Japan).

\section{Statistical analysis}

The data were presented as mean \pm SD. All statistical analysis was performed using GraphPad Prism 5.0 software. $P<0.05$ expresses that the differences were statistically significant.

\section{Results}

\section{Overexpression of HNF1A regulated the invasion power of ESCC cells.}


In our previous study, we successfully constructed ESCC cell lines TE1 and KYSE150 with overexpression protein of HNF1A. The expression levels of mRNA and protein of HNF1A were verified by RT-qPCR and WB assay. All results showed that HNF1A was still highly expressed in lentivirus transfected cell lines, and its levels of mRNA and protein were significantly higher than those in NC group (Fig. 1. a, b and c). In TE1 and KYSE150 cells overexpressing HNF1A, mRNA level was more than 2000 times $(P=0.032, P=0.009)$ and protein level was more than 2 times $(P=0.000, P=0.001)$. Our primary experiment found that HNF1A was involved in the invasion of ESCC cells. In this study, the invasion ability of TE1 and KYSE150 cells were clearly increased after ectopic expression of HNF1A $(P=0.025, P=0.023)$ (Fig. 1. $d$ and e). When TE1 and KYSE150 cells were irradiated, the invasion ability of NC group and HNF1A group observably enhanced $(P=0.011, P=0.006, P=0.014, P=0.006$, respectively), and the increasing level was much significant in HNF1A group than in NC group $(P=0.01, P=0.006)$.

\section{HNF1A interacted with HSPD1 in ESCC cells.}

In this paper, we paid more attention to explore mechanism of HNF1A affecting the invasion of ESCC cells. We used IP and liquid chromatography-tandem mass spectrometry (LC-MS/MS) assay to analyze HNF1A correlated proteins in TE1 cells. The differential protein bands were examined on the silver-stained SDS-PAGE compared with IgG control (Fig. 2, a). Next, we adopted mass spectrometry (MS) to identify differential protein bands (Report Number: R201901097). We had identified several bands proteins that possibly interact with HNF1A, such as HSPD1, hnRPA1/A3, and TCP1. HSPD1 protein was selected for subsequent research based on its score and protein banding localization.

Through GEPIA online database analysis, we obtained the association between HNF1A and HSPD1 in TCGA tumor tissues and normal tissues $(P<0.001)$ (Fig. 2. C). In order to clarify relationship between HNF1A and HSPD1, CO-IP technology was used to verify its function. Results showed that strongly confirmed interaction between HNF1A and HSPD1 (Fig. 2. b). The IF assay was used to detect the subcellular localization of HNF1A and HSPD1. Before IR, we discovered HNF1A protein expressed in nucleus, while HSPD1 protein ubiquitously existed in nucleus and cytoplasm. Moreover, HNF1A protein expression was enhanced and translocated from nucleus to cytoplasm after IR, which clearly demonstrated that HNF1A and HSPD1 co-localized in nucleus and cytoplasm (Fig. 2. d). This results again proved reciprocity between HNF1A and HSPD1.

\section{Radiation-induced cells reduced HSPD1 expression, which was not regulated by HNF1A.}

In order to illustrate how HNF1A and HSPD1 regulated each other, we employed WB experiment to verify. The HSPD1 protein expression was decreased in TE1 and KYSE150 cells after ectopic expression of HNF1A $(P=0.001, P=0.009)$. From this, we may consider that HNF1A negatively regulated HSPD1 protein expression. However, after IR 2h, HSPD1 protein in NC group reduced $(P=0.011, P=0.003)$, and 
the change trend in HNF1A group was not significant, and even a little bit higher. Notably, expression of HSPD1 protein in HNF1A group was higher than that in NC group after IR $(P=0.009, P=0.004)$ (Fig. 2. e). According to this results which HNF1A negatively regulated HSPD1 protein expression, the result was obviously contrary to it. Therefore, we had a novel discover that HNF1A was not involved in regulating HSPD1 expression, but their combination might play a pivotal role in tumor progression. In addition, our foregone study uncovered that the HNF1A expression reached its highest point after IR $2 \mathrm{~h}$, and the HSPD1 expression cut down the lowest point at $4 \mathrm{~h}$ after IR in our current research (Fig. 2. f). Based on the above, it was explicit that HSPD1 protein expression was decreased after IR, and it was not subject to HNF1A.

\section{The plasmid of HSPD1 knockdown was successfully screened and HSPD1 relieved radio-resistance from ectopic expression HNF1A.}

Based on these results, we hypothesized that HNF1A was not involved in regulating HSPD1 expression, but HNF1A needed HSPD1 as a molecular chaperone to perform its biological functions. Next, we adopted transwell experiment to verify whether absence of HSPD1 affect the phenomenon of HNF1A overexpression and promoted ESCC cells invasion ability. First, we learned from the GEPIA database that HSPD1 was widely high expressed in multifarious cancer types (Fig. 3. a), and HSPD1 expression was higher in ESCC tissues than in paracancerous tissues form TCGA database $(P<0.05)$ (Fig. 3. b). Using the GEPIA database to analyze the function of HSPD1 on survival of patients with ESCC, it revealed that patients with high expression of HSPD1 had poor OS $(H R=2.1, P=0.025)$. Therefore, we had reasons to speculate that HSPD1 might play an oncogene role in ESCC. Next, we transfected the knockdown plasmids of Sh-HSPD1 or Sh-NC into TE1 and KYSE150 cells by using lipofectamine 2000. These results disclosed that all knockdown plasmids inhibited HSPD1 expression at mRNA level and protein level (Fig. 3. d, e and f). ShRNA1 and ShRNA2 were more effective than ShRNA3 in HSPD1 protein knockdown by WB (Fig. 3. d, e). Meanwhile, the knockdown change of ShRNA1 and ShRNA2 were more significant at mRNA level (Fig. 3. f). Therefore, ShRNA1 and ShRNA2 were selected for subsequent experiments. Colony formation assay revealed that deletion of HSPD1 could enhance radiosensitivity of TE1 and KYSE150 cells (SER $=1.32$, SER $=1.33$ ), and overexpression of HNF1A could induce radio-resistance of TE1 and KYSE150 cells (SER $=0.64$, SER $=0.66$ ). Notably, knockout HSPD1 reversed that HNF1A promoted radioresistance in TE1 and KYSE150 cells (SER $=0.80$, SER $=0.89$, SER increasing compared with alone overexpression of HNF1A) (Fig. 3. g).

\section{Knockdown HSPD1 gene protein resulted to inhibit EMT process and affect the invasion ability in ESCC cells.}


After plasmid knockdown screening, HSPD1 effect on the invasion of ESCC cells was detected by transwell assay. These data showed that the invasion ability was significantly reduced before IR in HSPD1 knock-out TE1 and KYSE1 50 cells (shRNA1, $P=0.002, P=0.007$; ShRNA2, $P=0.004, P=0.044$, respectively) (Fig. 4. a, b). At the same time, invasion ability of all groups were increased after IR (NC, P = $0.000, P=0.015 ;$ shRNA1, $P=0.004, P=0.002$; ShRNA2, $P=0.000, P=0.007$, respectively) (Fig. 4. a, b). However, the invasion ability of ShRNA groups were decreased compared with NC group in TE1 and KYSE150 cells after IR (shRNA1, $P=0.000, P=0.014$; ShRNA2, $P=0.000, P=0.02$, respectively) (Fig. 4. a, b). Furthermore, the expression of EMT-related proteins was detected by WB. These results suggested that with or without IR, E-cadherin proteins were intensive in ShRNA group compared to NC group, but Ncadherin/Vimentin proteins were inverse (Fig. 5. a). In the meantime, E-cadherin proteins were depressed and $\mathrm{N}$-cadherin/Vimentin proteins were enhanced in all groups after IR (Fig. 5. a). Consequences indicated that the knockdown of HSPD1 gene protein reduced invasion ability via inhibiting the EMT process in ESCC cells.

\section{HSPD1 reversed increasing cell invasion ability of HNF1A overexpression by inhibited EMT process.}

Next, the HSPD1 knock-out effect on the invasion ability was observed in HNF1A overexpressing cells. We discovered that knockdown HSPD1 reversed the tendency of HNF1A to enhance cell invasion power (Fig. 4. c). Regardless of IR, HSPD1 knockout in HNF1A ectopic expressing cells resulted in cut down invasion ability (all P< 0.05) (Fig. 4. d). In addition, HSPD1 was knocked down in HNF1 Aoverexpressing cells, and total proteins of cells were extracted. These results revealed that E-cadherin proteins were improved in ShRNA group compared to control check (CK) and NC groups, while N-cadherin/Vimentin proteins were decreased with or without IR (Fig. 5. b).

\section{The exosomal secretion inhibitor GW4869 kept HSPD1 proteins inside cell after IR.}

Based on the results of above experimental, we concluded that HNF1A could not regulate HSPD1 expression, but HNF1A required the assistance of HSPD1 in regulating EMT and influenced cell invasion. On the other hand, radiation-induced decreased protein expression of HSPD1, but ectopic express HNF1A could keep from its decreasing. The regulating mechanism remained unclear still now. Recently, it had been reported that HSPD1 was distributed on exosome membrane and released into the tumor microenvironment, then affecting tumor cell production and progression(11). We used GW4869, an inhibitor of exosome secretion, to observe that irradiated may cause changes of HSPD1 protein in cells total protein or not. These results revealed that content of HSPD1 in GW4869 group was significantly higher than that in DMSO group before $I R(P=0.000, P=0.009)$ (Fig. 5. e, f). Moreover, intracellular HSPD1 protein in DMSO group was significantly reduced after IR $(P=0.002, P=0.006)($ Fig. $5 e, f)$. At the same time, HSPD1 protein in GW4869 group was higher than it in DMSO group $(P=0.013, P=0.032)$ 
(Fig. 5. e, f). The results indirectly demonstrated that HSPD1 protein was secreted via exosomes.

According to instructions of the exosome extraction kit, exosomes of TE1 cells were extracted and verified by TEM and WB assay. TEM showed that exosomes were successfully presented (Fig. 5. c). Positive markers Tsg101/Alix/CD9 proteins were checked by WB assay, while negative markers Calnexin protein was not detected (Fig. 5. d). It had been confirmed that exosomes were successfully extracted from supernatant of TE1 cells culture again.

\section{Ectopic expression HNF1A reduced HSPD1 secretion by exosomes outside cells after IR.}

Notably, all results also revealed that the presence of HSPD1 in exosomes, which directly proved HSPD1 protein could be secreted into extracellular environment through exosomes (Fig. 5. d). After IR, trendency HSPD1 protein expression in GW4869 group was consistent with those in HNF1A group. We hypothesized that ectopic expression HNF1A reduced releasing of HSPD1 protein in exosomes, resulting in HSPD1 stop in cells and assisting HNF1A to play biological functions. Next, we employed WB verifying our conjecture. The content of HSPD1 in exosomes significantly decreased with or without IR after overexpression of HNF1A ( $P=0.102, P=0.006$ ) (Fig. $5 g$ ). After IR, HSPD1 protein content of exosomes in NC group was significantly increased $(P=0.005)$, but there were no statistically significant difference in HNF1A group ( $P$ $=0.197$ ) (Fig. 5. g). Our results affirmed that radiation-induced HSPD1 from exosomes pathway were largely released outside cells and that ectopic expression HNF1A could weaken this phenomenon.

\section{Discussion}

This study investigated biological function of HNF1A and its possible molecular mechanisms in ESCC. In order to better understand role of HNF1A in ESCC, this study emphatically analyzed that HNF1A regulated radiation resistance and invasion ability of ESCC. In addition, the present study provided a unique perspective to reveal that cells with overexpression of HNF1A may elude the anti-tumor immune response via inhibiting the extracellular release of HSPD1 (releasing by exosomes), and ultimately leading to radioresistance of ESCC cells.

Based on existing studies, the combination of HNF1A and HSPD1 in regulating radiosensitivity of ESCC was novel. HNF1A played an oncogenic role in a variety of tumors, such as pancreatic cancer, hepatocellular carcinoma and gastric cancer $(5,6,13)$. Our results suggested that ectopic expression HNF1A promoted radio-resistance of ESCC to release potential oncogenic ability. HSPD1 had been identified as a high-quality prognostic biomarker in multiple cancers, such as non-small cell lung cancer, ovarian cancer, metastatic colorectal cancer, gastric cancer, hepatocellular carcinoma(14-21). At the same time, HSPD1 inhibitors and antibodies had shown remarkable efficacy in basic experiments(2224). TGCA database showed that HSPD1 was highly expressed in multifarious tumors, and ESCC patients with high HSPD1 expression had poor prognosis. These results suggested that HSPD1 was an oncogene in ESCC. Although HNF1A and HSPD1 played a carcinogenic role in ESCC, it has not been 
reported a connection between them in previous studies. We revealed for the first time that HNF1A binded to HSPD1, but HNF1A did not regulate the HSPD1 protein expression.

Further studies demonstrated that depletion of HSPD1 weakened radio-resistance in ESCC cells with ectopic expression HNF1A. There were evidences that HSPD1 is involved in maintaining normal protein homeostasis and protein denaturation repair $(9,25)$. We speculated that HSPD1 was consumed with modifying new abundant proteins which maintain cell function after ectopic expression of HNF1A. Furthermore, after radiation-induced denaturation of proteins in cells, HSPD1 was expended to repair denatured protein. At the same time, overexpression of HNF1A reduced radiation-induced the occurrence of protein denaturation, thus abating the consumption of HSPD1. Finally, we preliminarily concluded that HNF1A and HSPD1 were combined, but there was no regulatory relationship.

In this study, ectopic expression HNF1A activated EMT to promote invasion of ESCC cells, which required the assistance of HSPD1 protein. A study reported that miR-484 inhibited the expression of MMP14/HNF1A in vitro and in vivo to weaken EMT process, leading to recede invasion of cervical cancer cells(26). Similar results have been obtained in gastric cancer(5). At the same time, knockout of HSPD1 resulted in reduced invasion by impairing the EMT process in oral squamous cell carcinoma, and was be related to prognosis(27). In addition, activating EMT not only regulated the cell invasion ability, but were also associated with cell proliferation, apoptosis and drug resistance(28). Our results showed that HSPD1 assisted HNF1A to influence the ESCC progression by regulating EMT.

There were substantial evidences that the exosomal inhibitor GW4869 inhibited exosome secretion in multifarious tumor and normal cells(29-32). Our data showed that TE1 and KYSE150 cells treated with GW4869 increased the amount of HSPD1 protein inside the cells. This indirectly indicated that HSPD1 could be secreted extracellular through exosomes. Exosomal protein markers Tsg101/Alix/CD9 bands were detected, and their presence represented the successful extraction of exosomes $(33,34)$. Next, exosomes were extracted from TE1 cells. We found that the content of HSPD1 protein in exosomes increased after IR. It was consistent with other results, in anticancer drug-treated liver cancer cells, ascites of malignant gastric cancer patients and heat-shocked mouse B lymphoma cells released exosomes including abundant HSP60 proteins, which can effectively induce anti-tumor immune responses(35-37). These studies had confirmed that tumor cells stimulated by physical or chemical drugs will secrete exosomes containing a large of HSP60 proteins, thus inducing anti-tumor immune response. We initially demonstrated that radiation-induced secretion of exosomes contained rich HSPD1 proteins in ESCC cells, and overexpression of HNF1A could counteract this induction.

Based on above results, we analyzed potential molecular mechanisms which combination of HNF1A and HSPD1 affected invasion ability and radiosensitivity of ESCC. On the one hand, the mechanism might be that ectopic express HNF1A reduced radiation-induced protein degeneration. It resulted in less consumption of HSPD1 involved in protein repair and had more opportunities to help folding and modification of new proteins, thus promoting ESCC progression. On the other hand, we hypothesized that 
HNF1A prevented the extracellular release of HSPD1 via exosomes after IR, which weakened the initiation of anti-tumor immune responses, thereby leading to radioresistance in ESCC.

\section{Conclusions}

In conclusion, we confirmed HNF1A was assisted with HSPD1 and promoted the invasion of ESCC cells by driving EMT, and initially revealed that HNF1A reduced radiation-induced exosomal release of HSPD1, leading to radioresistance of ESCC cells. However, whether the increase of HSPD1 protein in exosomes induced by radiation was related to anti-tumor immune response, it remained to be clarified in subsequent experiments. Available data and results encourage creative design of novel cancer therapy strategies that combine targeting HNF1A and HSPD1 genes to enhance radiosensitivity in ESCC. HSPD1 in exosomes was mightily to be a novel promising predictive and prognostic biomarker.

\section{Declarations}

\section{Acknowledgments}

Not applicable.

\section{Authors' contributions}

The subject design was completed by NZ, XZ and SZ. The operation of the experiment was carried out by NZ, JD and SL. Data analysis was performed by NZ, JD, YL, JX and YZ. Article written by NZ and XZ. The final data review and article review by SZ. All authors read and approved the final manuscript.

\section{Funding}

The present study was supported by the National Natural Science Foundation of China (no. 81872456), Natural Science Foundation of Hebei Province (no. H2020206583) and a grant from the Education Department of Hebei Province (no. CXZZBS2021078).

\section{Availability of data and materials}

The data and materials in the current study are available from the corresponding author on reasonable request.

\section{Ethics approval and consent to participate}

Not applicable. 


\section{Consent for publication}

Not applicable.

\section{Competing interests}

The authors have no conflicts of interest.

\section{Author details}

${ }^{1}$ Department of Radiation Oncology, The Fourth Hospital of Hebei Medical University, Shijiazhuang, Hebei 050011, P.R. China.

\section{References}

1. F B, J F, I S, RL S, LA T, A J. Global cancer statistics 2018: GLOBOCAN estimates of incidence and mortality worldwide for 36 cancers in 185 countries. CA Cancer J Clin. 2018;68(6):394-424.

2. Liang H, Fan JH, Qiao YL. Epidemiology, etiology, and prevention of esophageal squamous cell carcinoma in China. Cancer Biol Med. 2017;14(1):33-41.

3. Ma W-J, Zhang Q-N, Shi S-Z, Zhang X-Y, Zhao Y, Tian J-H, et al. Preoperative chemoradiation may be more effective for esophageal squamous cell carcinoma compared with adenocarcinoma: results from 15 randomized controlled trials of 2,250 patients. Transl cancer res. 2018;7(6):1421-30.

4. Lau H, Ng N, Loo L, Jasmen J, Teo A. The molecular functions of hepatocyte nuclear factors - In and beyond the liver. Journal of hepatology. 2018;68(5):1033-48.

5. Ma P, Li L, Liu F, Zhao Q. HNF1A-Induced IncRNA HCG18 Facilitates Gastric Cancer Progression by Upregulating DNAJB12 via miR-152-3p. OncoTargets therapy. 2020;13:7641-52.

6. Fan Z, Fan K, Deng S, Gong Y, Qian Y, Huang Q, et al. HNF-1a promotes pancreatic cancer growth and apoptosis resistance via its target gene PKLR. Acta biochimica et biophysica Sinica. 2020;52(3):24150.

7. Abel E, Goto M, Magnuson B, Abraham S, Ramanathan N, Hotaling E, et al. HNF1A is a novel oncogene that regulates human pancreatic cancer stem cell properties. eLife. 2018;7.

8. Lin K, Lin B, Lian I, Mestril R, Scheffler I, Dillmann W. Combined and individual mitochondrial HSP60 and HSP10 expression in cardiac myocytes protects mitochondrial function and prevents apoptotic cell deaths induced by simulated ischemia-reoxygenation. Circulation. 2001;103(13):1787-92.

9. Rodriguez A, Von Salzen D, Holguin BA, Bernal RA. Complex Destabilization in the Mitochondrial Chaperonin Hsp60 Leads to Disease. Front Mol Biosci. 2020;7:159.

10. Caruso Bavisotto C, Alberti G, Vitale AM, Paladino L, Campanella C, Rappa F, et al. Hsp60 Posttranslational Modifications: Functional and Pathological Consequences. Front Mol Biosci. 2020;7:95. 
11. Caruso Bavisotto C, Cappello F, Macario A, Conway de Macario E, Logozzi M, Fais S, et al. Exosomal HSP60: a potentially useful biomarker for diagnosis, assessing prognosis, and monitoring response to treatment. Expert review of molecular diagnostics. 2017;17(9):815-22.

12. Campanella C, D'Anneo A, Marino Gammazza A, Caruso Bavisotto C, Barone R, Emanuele S, et al. The histone deacetylase inhibitor SAHA induces HSP60 nitration and its extracellular release by exosomal vesicles in human lung-derived carcinoma cells. Oncotarget. 2016;7(20):28849-67.

13. Zhan X, Zhao A. Transcription factor FOXA3 promotes the development of Hepatoblastoma via regulating HNF1A, AFP, and ZFHX3 expression. Journal of clinical laboratory analysis. 2020:e23686.

14. Ağababaoğlu İ, Önen A, Demir A, Aktaş S, Altun Z, Ersöz H, et al. Chaperonin (HSP60) and annexin-2 are candidate biomarkers for non-small cell lung carcinoma. Medicine. 2017;96(6):e5903.

15. Fucarino A, Pitruzzella A. Role of HSP60/HSP10 in Lung Cancer: Simple Biomarkers or Leading Actors? Journal of oncology. 2020;2020:4701868.

16. Vocka M, Langer D, Fryba V, Petrtyl J, Hanus T, Kalousova M, et al. Novel serum markers HSP60, CHI3L1, and IGFBP-2 in metastatic colorectal cancer. Oncology letters. 2019;18(6):6284-92.

17. Tang Y, Yang Y, Luo J, Liu S, Zhan Y, Zang H, et al. Overexpression of HSP10 correlates with HSP60 and $\mathrm{Mcl}-1$ levels and predicts poor prognosis in non-small cell lung cancer patients. Cancer biomarkers. 2020.

18. Chen Y, Li X, Shao S. The Clinical Value of HSP60 in Digestive System Cancers: a Systematic Review and Meta-Analysis. Clinical laboratory. 2019;65(10).

19. Harper A, Fletcher N, Fan R, Morris R, Saed G. Heat Shock Protein 60 (HSP60) Serves as a Potential Target for the Sensitization of Chemoresistant Ovarian Cancer Cells. Reproductive sciences. 2020;27(4):1030-6.

20. Huang Y, Lin K, Yu J, Wu T, Lee W, Chao C, et al. Targeting HSP60 by subcutaneous injections of jetPEI/HSP60-shRNA destabilizes cytoplasmic survivin and inhibits hepatocellular carcinoma growth. Molecular carcinogenesis. 2018;57(9):1087-101.

21. Zhang J, Zhou X, Chang H, Huang X, Guo X, Du X, et al. Hsp60 exerts a tumor suppressor function by inducing cell differentiation and inhibiting invasion in hepatocellular carcinoma. Oncotarget. 2016;7(42):68976-89.

22. Meng Q, Li B, Xiao X. Toward Developing Chemical Modulators of Hsp60 as Potential Therapeutics. Frontiers in molecular biosciences. 2018;5:35.

23. Venkatesh S, Suzuki C. HSP60 Takes a Hit: Inhibition of Mitochondrial Protein Folding. Cell chemical biology. 2017;24(5):543-5.

24. Stevens M, Abdeen S, Salim N, Ray A, Washburn A, Chitre S, et al. HSP60/10 chaperonin systems are inhibited by a variety of approved drugs, natural products, and known bioactive molecules. Bioorganic medicinal chemistry letters. 2019;29(9):1106-12.

25. Tang $H$, Teng $R$, Zhao $X$, Wang $X, X u L$, Deng $H$, et al. Isotope tracing assisted metabolic profiling: Application to understanding HSP60 silencing mediated tumor progression. Analytica chimica acta. 2019;1047:93-103. 
26. Hu Y, Wu F, Liu Y, Zhao Q, Tang H. DNMT1 recruited by EZH2-mediated silencing of miR-484 contributes to the malignancy of cervical cancer cells through MMP14 and HNF1A. Clinical epigenetics. 2019;11(1):186.

27. Kang B, Shu C, Chao J, Lee C, Fu T, Liou H, et al. HSPD1 repressed E-cadherin expression to promote cell invasion and migration for poor prognosis in oral squamous cell carcinoma. Scientific reports. 2019;9(1):8932.

28. Tiwari N, Gheldof A, Tatari M, Christofori G. EMT as the ultimate survival mechanism of cancer cells. Seminars in cancer biology. 2012;22(3):194-207.

29. Faict S, Muller J, De Veirman K, De Bruyne E, Maes K, Vrancken L, et al. Exosomes play a role in multiple myeloma bone disease and tumor development by targeting osteoclasts and osteoblasts. Blood cancer journal. 2018;8(11):105.

30. Essandoh K, Yang L, Wang X, Huang W, Qin D, Hao J, et al. Blockade of exosome generation with GW4869 dampens the sepsis-induced inflammation and cardiac dysfunction. Biochimica et biophysica acta. 2015;1852(11):2362-71.

31. Liu X, Lu Y, Xu Y, Hou S, Huang J, Wang B, et al. Exosomal transfer of miR-501 confers doxorubicin resistance and tumorigenesis via targeting of BLID in gastric cancer. Cancer letters. 2019;459:122-34.

32. Kulshreshtha A, Ahmad T, Agrawal A, Ghosh BJTJoa. Proinflammatory role of epithelial cell-derived exosomes in allergic airway inflammation. J Allergy Clin Immunol. 2013;131(4):1194-203, 203.e1-14.

33. Zhang C, Zhu Z, Gao J, Yang L, Dang E, Fang H, et al. miR-375-3pPlasma exosomal regulates mitochondria-dependent keratinocyte apoptosis by targeting XIAP in severe drug-induced skin reactions. Sci Transl Med. 2020;12(574).

34. Hoshino A, Kim H, Bojmar L, Gyan K, Cioffı M, Hernandez J, et al. Extracellular Vesicle and Particle Biomarkers Define Multiple Human Cancers. Cell chemical biology. 2020;182(4):1044-61.e18.

35. Lv L, Wan Y, Lin Y, Zhang W, Yang M, Li G, et al. Anticancer drugs cause release of exosomes with heat shock proteins from human hepatocellular carcinoma cells that elicit effective natural killer cell antitumor responses in vitro. J Biol Chem 2012;287(19):15874-85.

36. Zhong H, Yang Y, Ma S, Xiu F, Cai Z, Zhao H, et al. Induction of a tumour-specific CTL response by exosomes isolated from heat-treated malignant ascites of gastric cancer patients. Int $\mathrm{J}$ Hyperthermia 2011;27(6):604-11.

37. Chen W, Wang J, Shao C, Liu S, Yu Y, Wang Q, et al. Efficient induction of antitumor T cell immunity by exosomes derived from heat-shocked lymphoma cells. Eur J Immunol. 2006;36(6):1598-607.

\section{Figures}


a
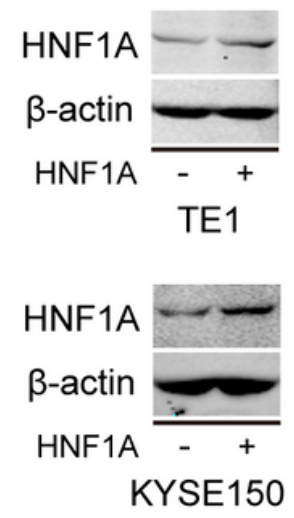

d
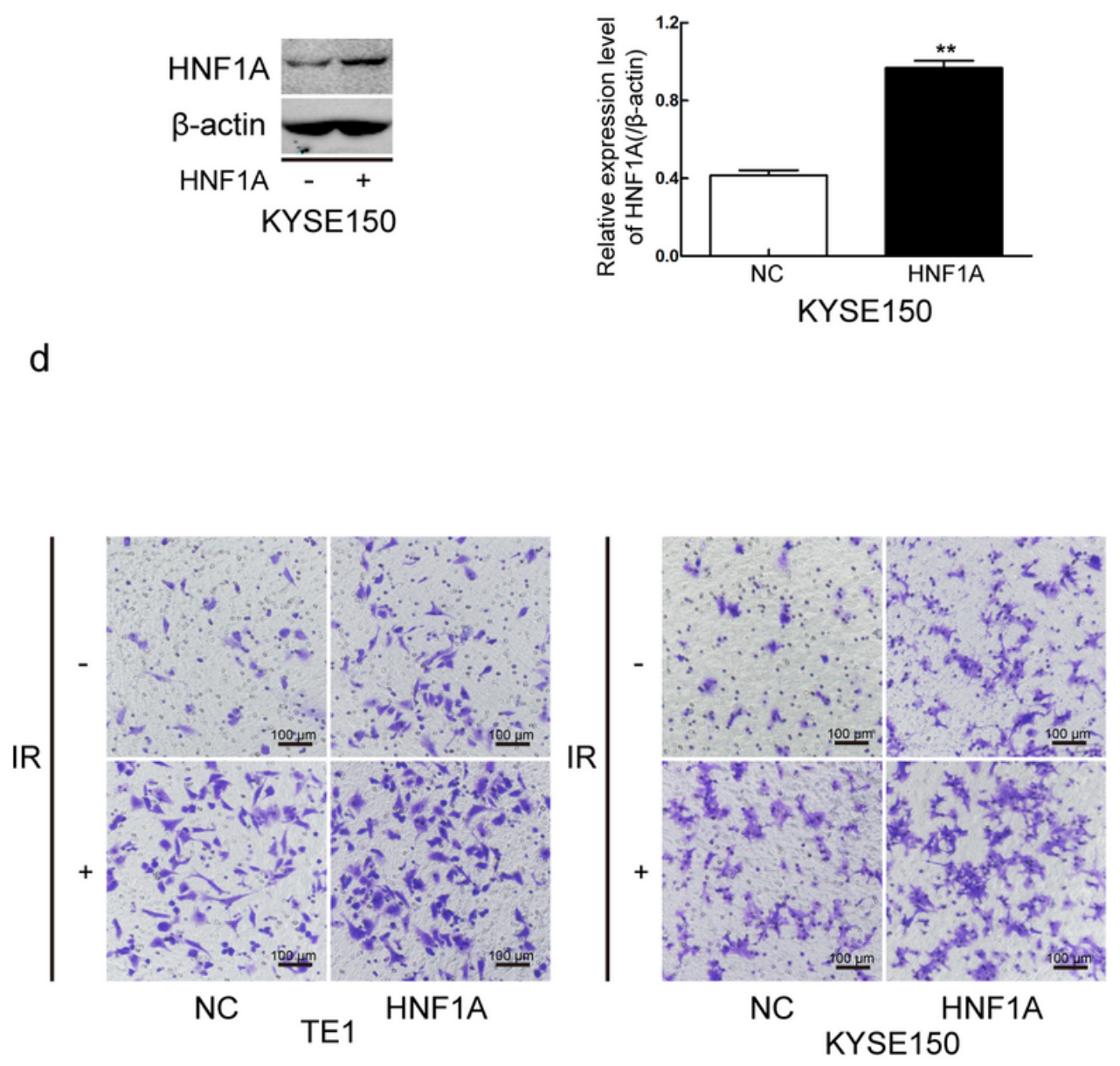

C
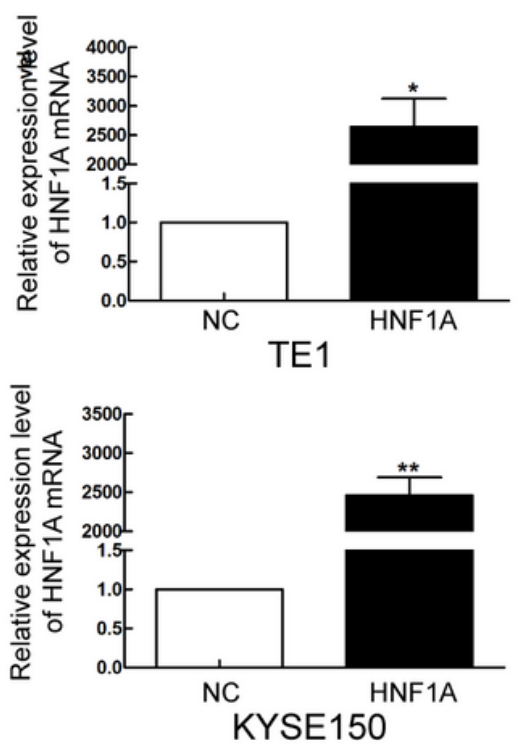

e
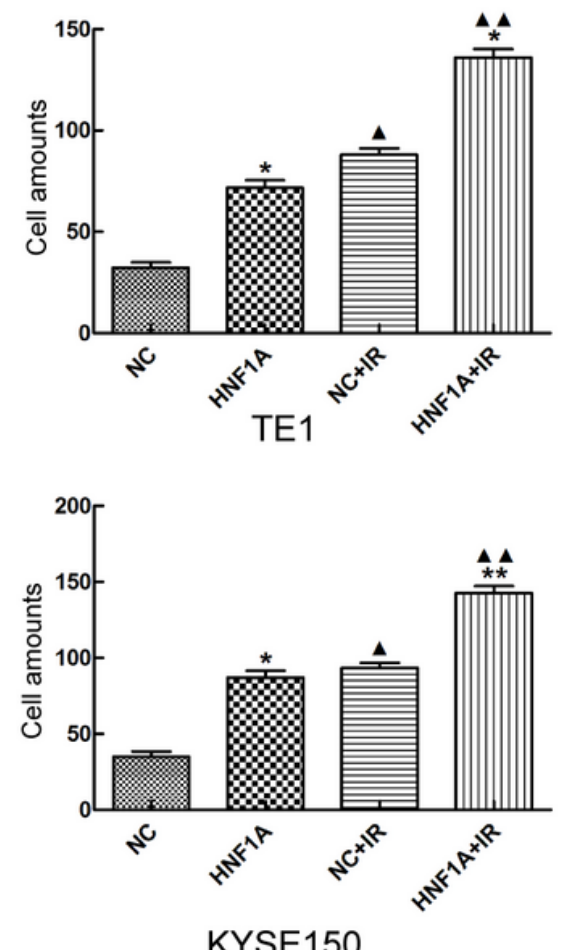

\section{Figure 1}

Overexpression of HNF1A promoted invasion of TE1 and KYSE150 cells. (a, b)WB assay was used to detect HNF1A protein expression in TE1 and KYSE150 cell lines. (c)Expression of HNF1A mRNA was detected by RT-qPCR. These results indicated that ectopic expression HNF1A cells were successfully built. (d, e)HNF1A overexpression enhanced invasion ability of ESCC cells by the transwell assay (magnification 200x). The error bars represent the standard deviation. ${ }^{*} p<0.05, * \star p<0.01, * \star * p<0.001$ compared with the NC group; $\mathbf{\Delta} p<0.05, \mathbf{\Delta} \mathbf{\Delta} p<0.01$ compared with the corresponding unirradiated group. 

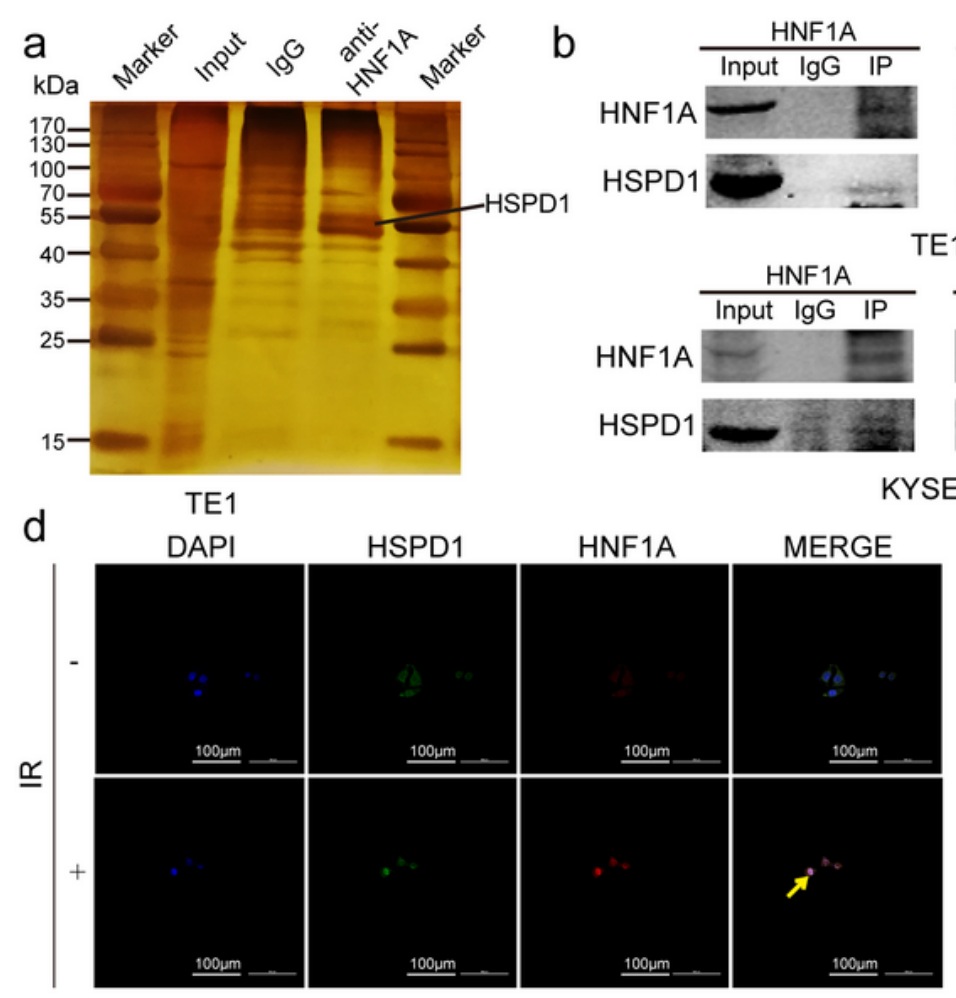

TE1

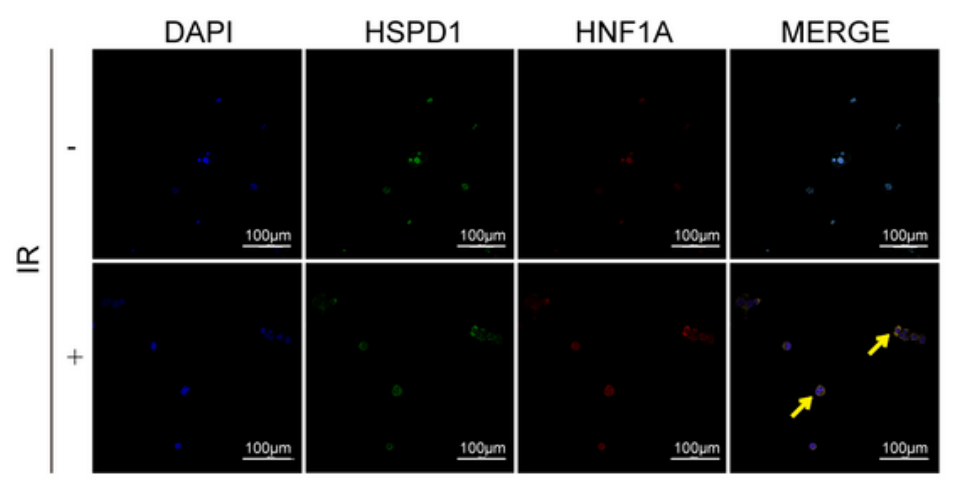

KYSE150

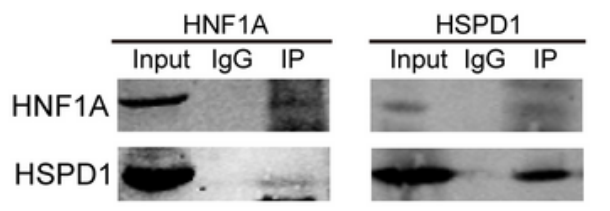

TE1

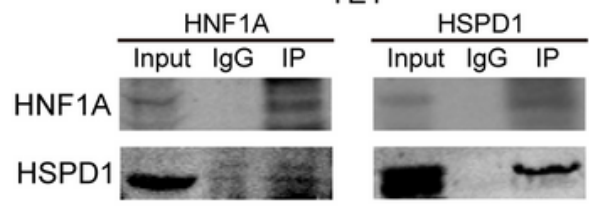

KYSE150
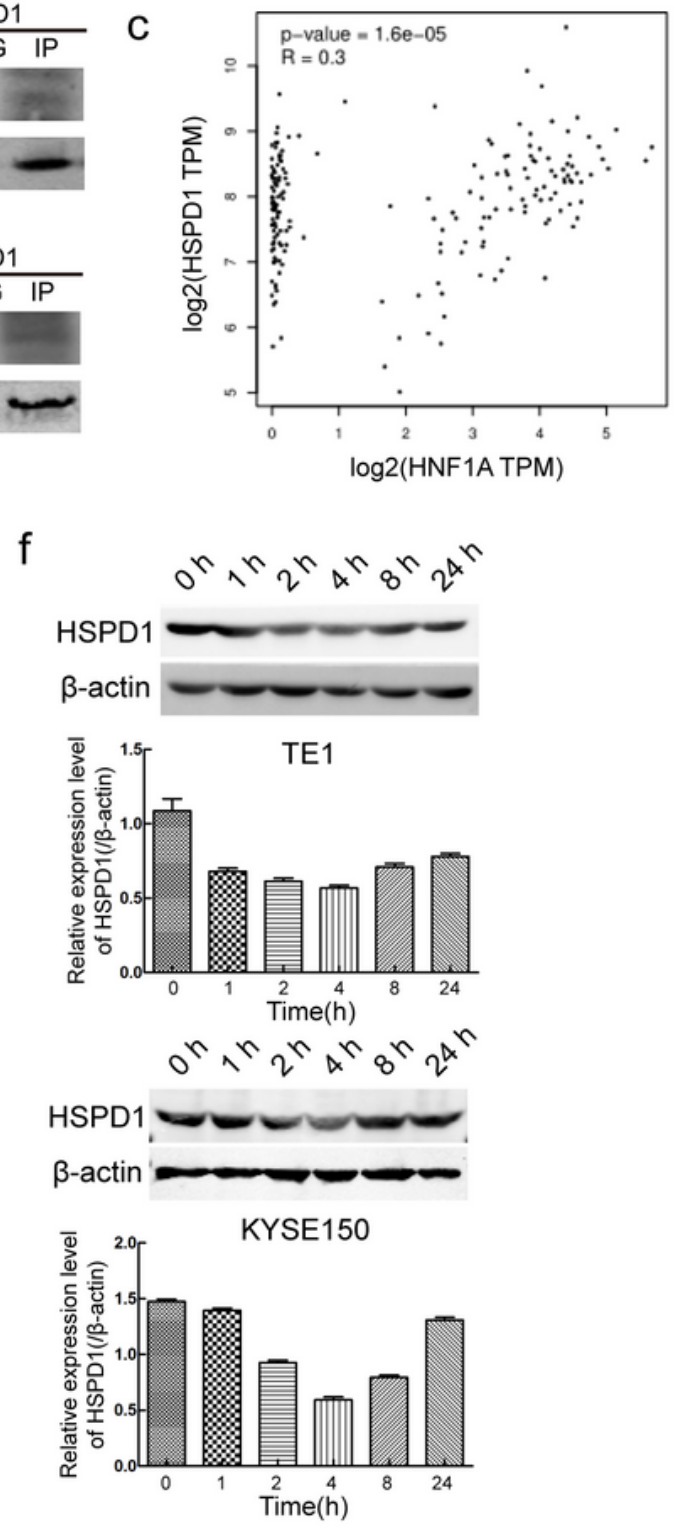

e
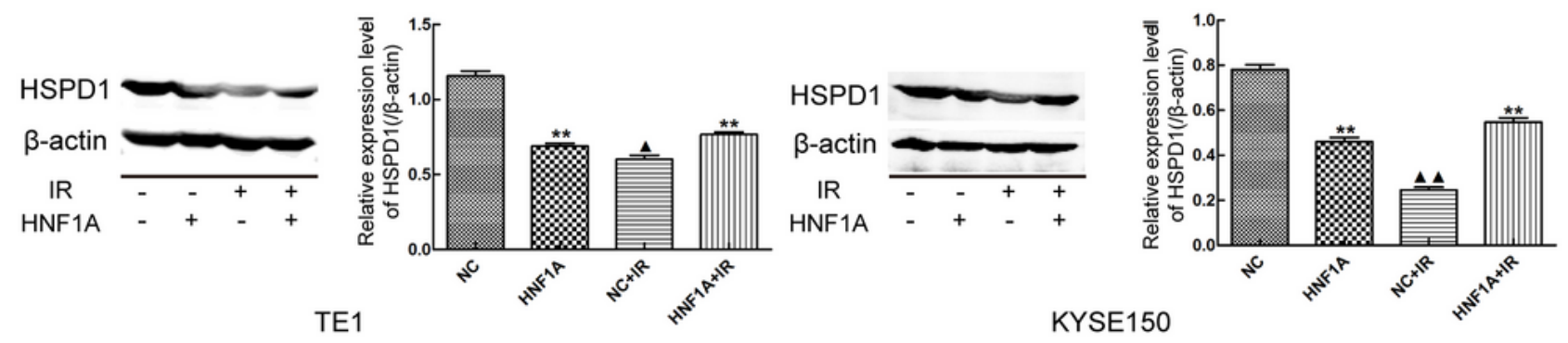

\section{Figure 2}

There was an interaction between HNF1A and HSPD1, and relationship between HSPD1 protein expression and time after irradiation. (a)The cells lysates were treated with anti-lgG antibody (IgG) and anti-HNF1A antibody (IP). Differential bands binding to HNF1A and IgG antibodies were detected and identified by LC-MS/MS in the silver stained sodium dodecyl sulfate-polyacrylamide gel electrophoresis.

(b)The interaction between HNF1A and HSPD1 was confirmed by CO-IP assay. (c)The correlation of 
HNF1A and HSPD1 in tumor tissues and paracancerous tissues were obtained from the TCGA database. (d)We observed Subcellar location of HNF1A and HSPD1 by IF assays. (e)Overexpression HNF1A effect on HSPD1 protein with or without IR in TE1 and KYSE150 cells were detected by WB. (f)After IR $4 \mathrm{~h}$, HSPD1 protein expression decreased to the lowest by WB assay. The error bars represent the standard deviation. ${ }^{* *} p<0.01$ compared with the NC group; $\boldsymbol{\Delta} p<0.05, \boldsymbol{\Delta} \mathbf{\Delta} p<0.01$ compared with the corresponding unirradiated group.

a

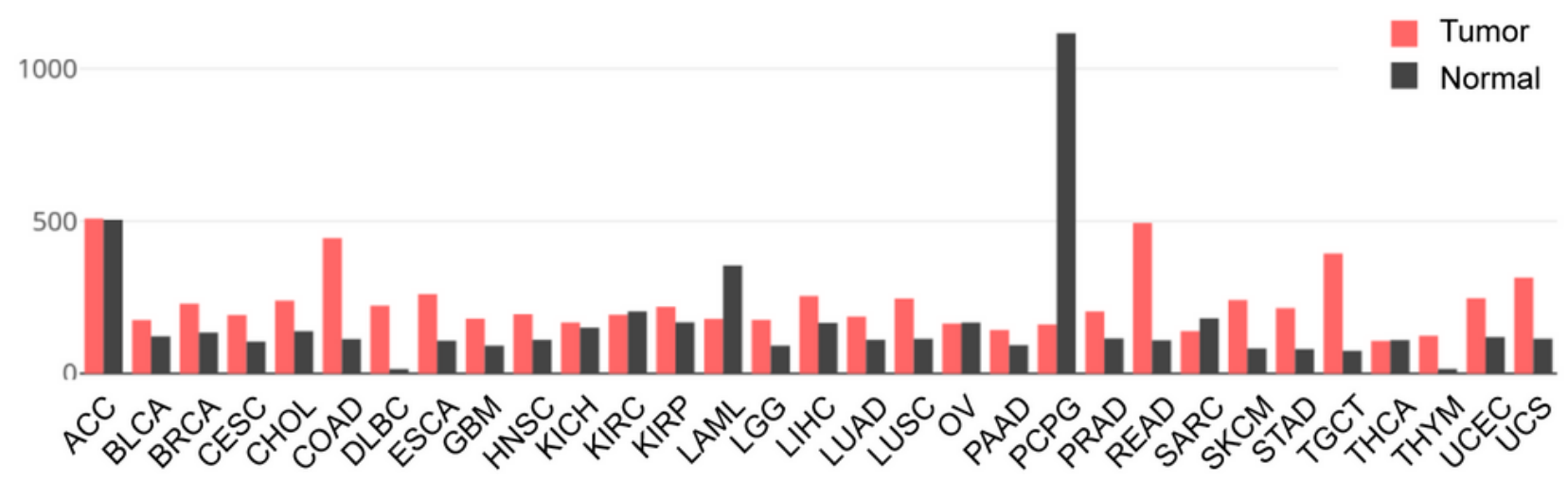

b

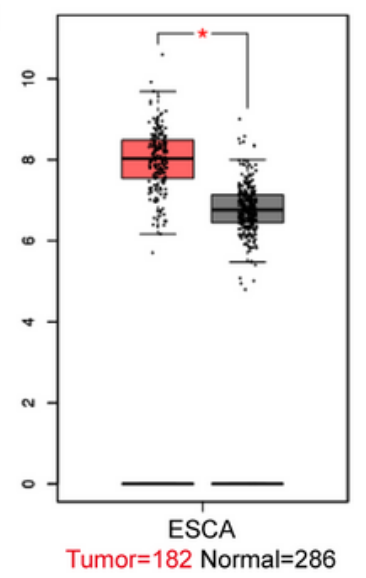

$$
\text { e }
$$
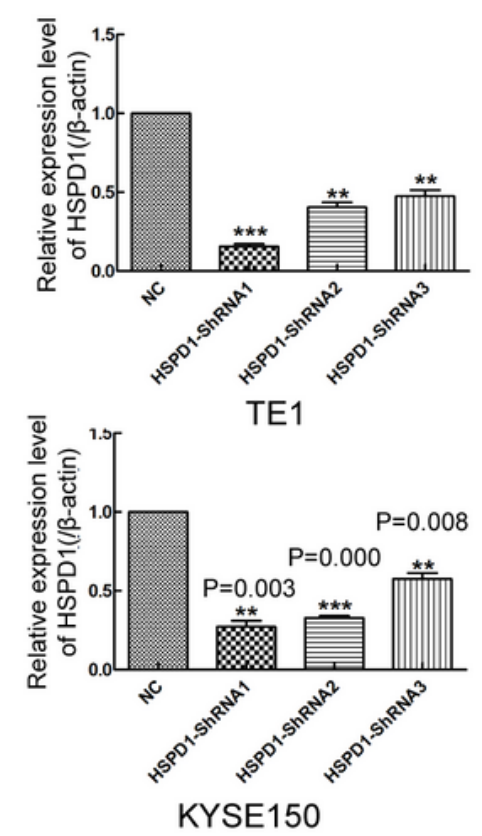

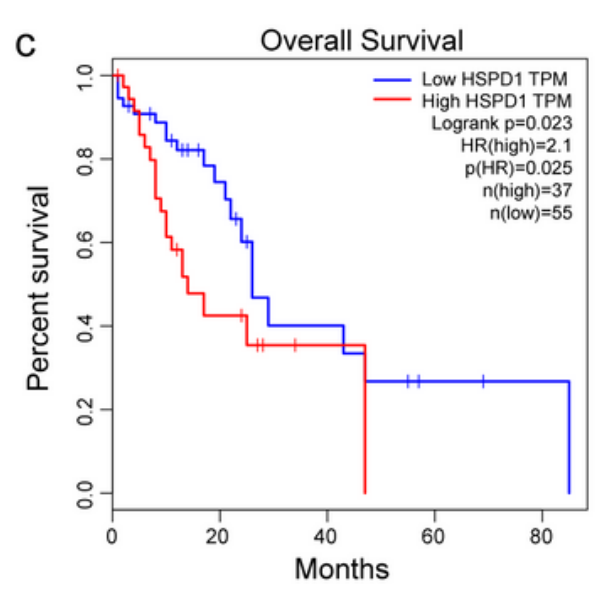

f

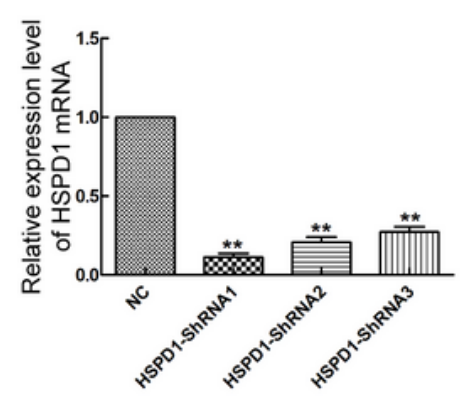

TE1

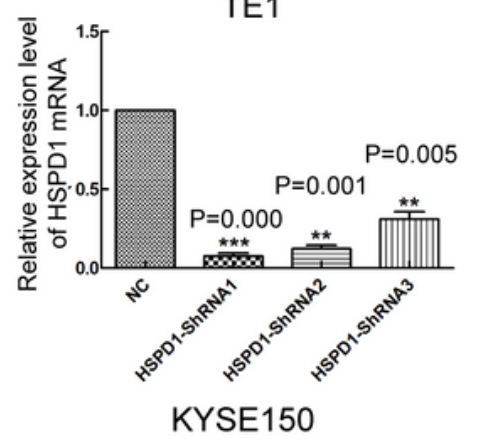

d

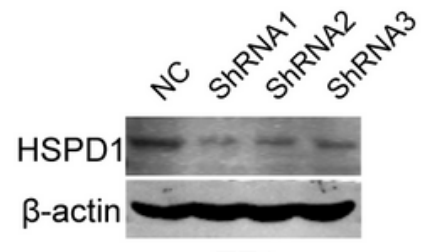

TE1

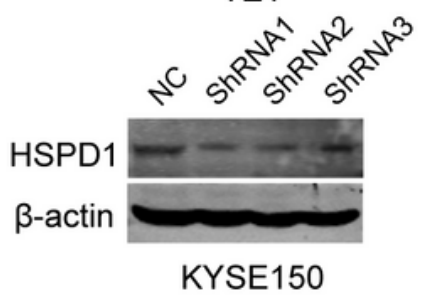

g
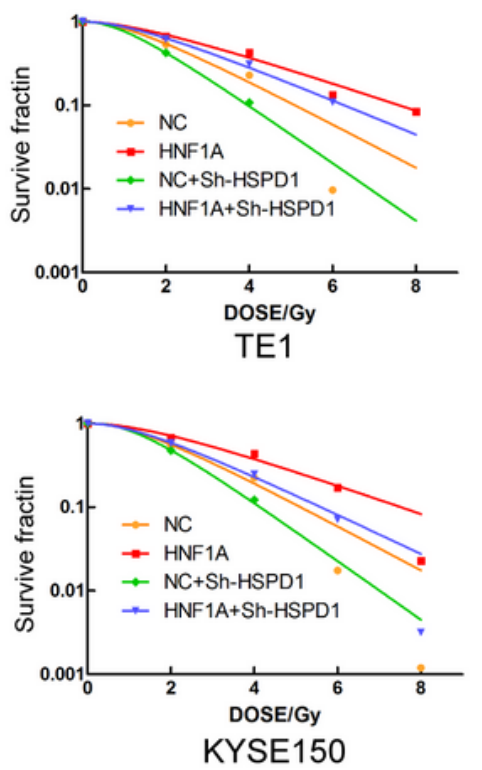

Figure 3 
The relationship between HSPD1 expression level and prognosis of patients with ESCC. (a)The expression of HSPD1 in various types of cancer were analyzed online by GEPIA. (b)The expression level of HSPD1 in tumor tissue and paracancerous tissues. (c)Patients with high HSPD1 expression level had a poor survival in ESCC. (d, e and f)The TE1 and KYSE150 cells were transfected with Sh-NC and ShHSPD1 plasmids. HSPD1 protein expression level was reduced after HSPD1 knockdown by WB assay. The mRNA expression of HSPD1 was detected by RT-qPCR. (g) Survival curve was plotted by colony formation assay. The error bars represent the standard deviation. ${ }^{\star \star} p<0.0, * \star \star p<0.001$ compared with the NC group. 
a

IR

$+$

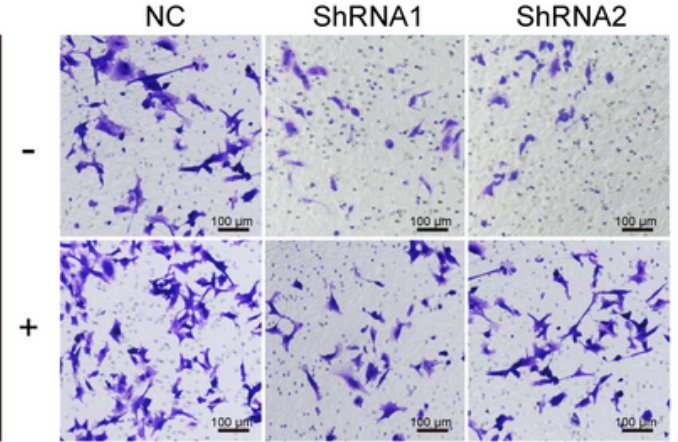

TE1

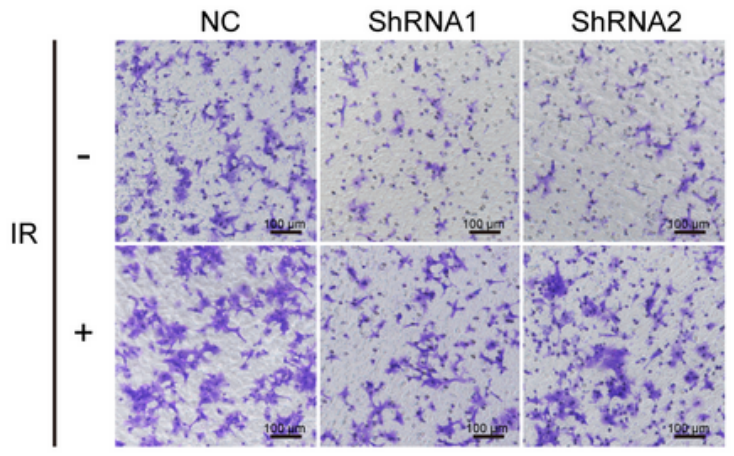

KYSE150

c

IR

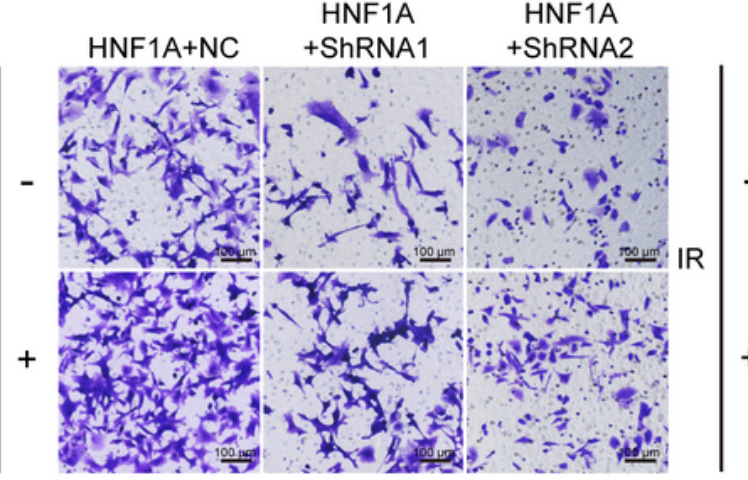

TE1

d

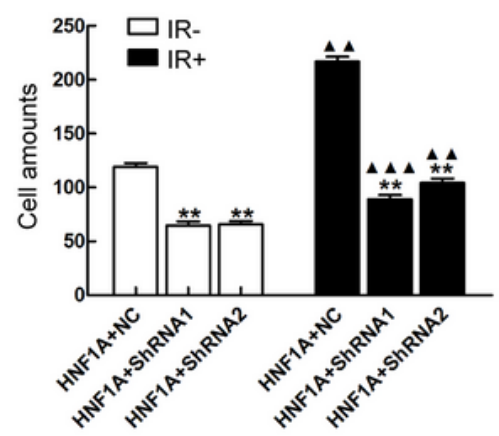

TE1 b

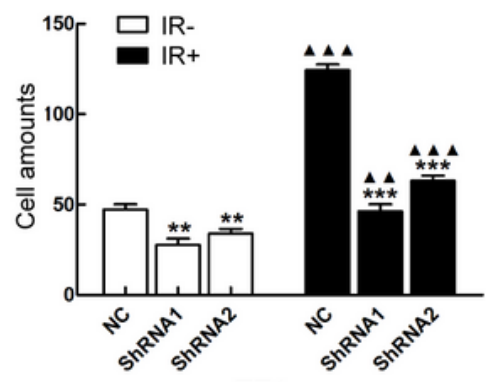

TE1
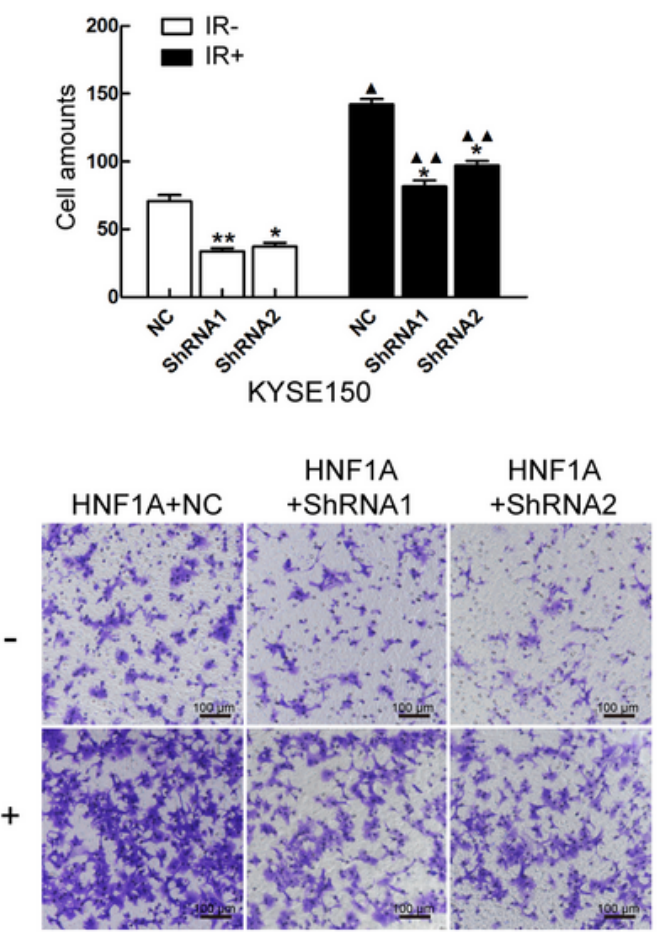

KYSE150

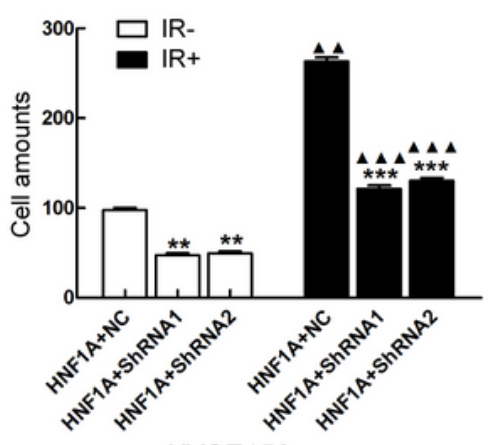

KYSE150

Figure 4

HSPD1 reversed that ectopic expression HNF1A promoted invasion ability of TE1 and KYSE150 cells.

(a)Sh-HSPD1 plasmid effect on invasion ability of TE1 and KYSE150 cells (magnification 200x). (b)The bar chart showed that number of cells passed through transwell chamber coated with matrigel matrix. (c)In TE1 and KYSE150 cells with stable overexpression of HNF1A, Sh-HSPD1 and Sh-NC plasmids were transfected into cells by lipofectamine 2000. Deletion of HSPD1 gene downregulated cell invasion power 
(magnification 200x). (d)Number of cells traversed matrigel matrix coated transwell chamber by the bar chart. The error bars represent the standard deviation. $* p<0.05,{ }^{\star *} p<0.01, * \star * p<0.001$ compared with the NC group; $\boldsymbol{\Delta} p<0.05, \boldsymbol{\Delta} \mathbf{\Delta} p<0.01, \boldsymbol{\Delta} \mathbf{\Delta} \boldsymbol{\Delta} p<0.001$ compared with the corresponding unirradiated group.

a
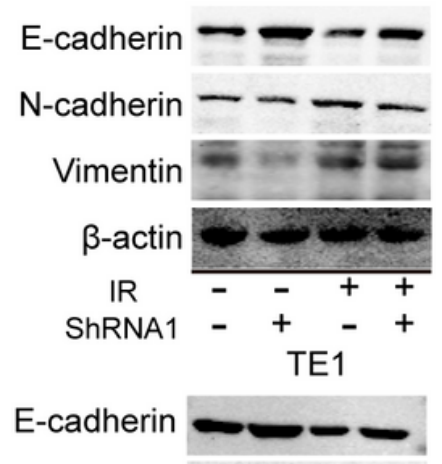

$\mathrm{N}$-cadherin $-\cdots-$

Vimentin

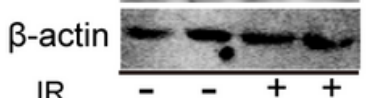

IR ShRNA e

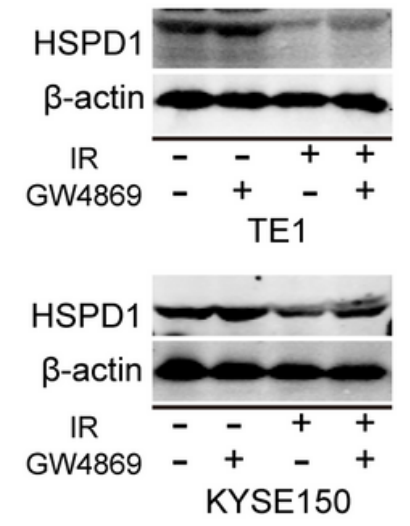

b
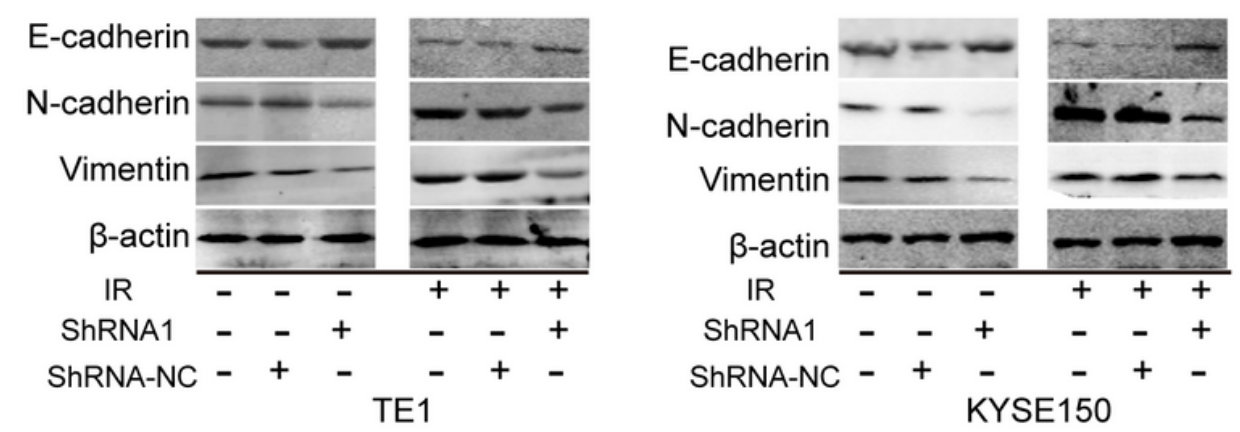

d

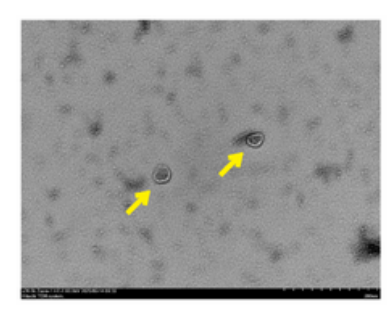

TE1-Exos

f

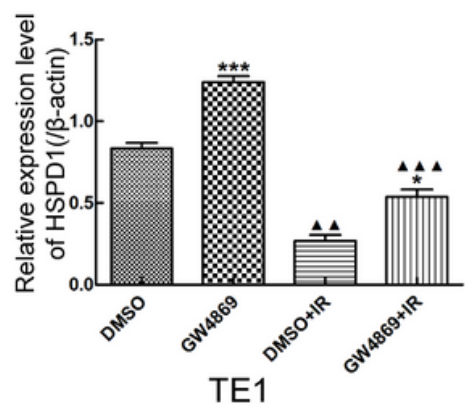

g

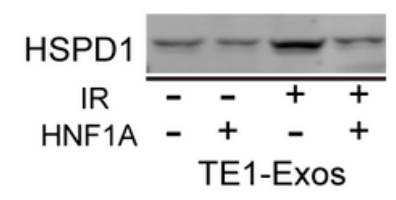

$\mathrm{h}$

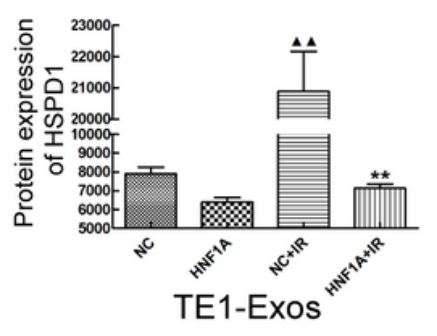

\section{Figure 5}

HSPD1 regulated EMT process and affected the invasion ability of ESCC cells. The content of HSPD1 in exosomes extracted from TE1 cell culture supernatant was depended on HNF1A or IR. (a)E-cadherin/Ncadherin/Vimentin protein expression was detected after cells treated by Sh-HSPD1 and Sh-NC plasmids. (b)When TE1 and KYSE150 cells overexpress HNF1A, the content of EMT-related proteins was observed by WB assay. (c)Exosomal morphology was observed by transmission electron microscopy (magnification 70,000x). (d) WB experiment was used to observe Alix/Tsg101/CD9/Calnexin/HSPD1 protein expression in exosomes were extracted from TE1 supernatant. $(e, f)$ The changes of HSPD1 expression protein content in total protein were detected by WB assay when cells were intervened with DMSO or GW4869. $(\mathrm{g}, \mathrm{h})$ The exosomes were extracted from TE1 cell culture supernatant of HNF1A group and NC group. The error bars represent the standard deviation. ${ }^{*} p<0.05,{ }^{\star \star} p<0.01,{ }^{\star \star \star} p<0.001$ 
compared with the NC group; $\boldsymbol{\Delta} p<0.05, \boldsymbol{\Delta} \mathbf{\Delta} p<0.01, \boldsymbol{\Delta} \mathbf{\Delta} \boldsymbol{\Delta} p<0.001$ compared with the corresponding unirradiated group. 